\title{
Natalizumab for Achieving Relapse-Free, T1 Gadolinium-Enhancing-Lesion-Free, and T2 Lesion- Free Status in Japanese Multiple Sclerosis Patients: A Phase 2 Trial Subanalysis
}

Takahiko Saida $\cdot$ Jun-Ichi Kira $\cdot$ Shuji Kishida $\cdot$ Takashi Yamamura •

Nobuhisa Ohtsuka • Qunming Dong · J. T. Tibung

Received: October 10, 2016 / Published online: January 11, 2017

(C) The Author(s) 2017. This article is published with open access at Springerlink.com

\section{ABSTRACT}

Introduction: In a phase 2 trial of natalizumab in Japanese patients with relapsing-remitting multiple sclerosis (RRMS), treatment-related changes in relapses, brain lesions, and disability worsening were found to be comparable with those observed in the phase 3 studies of natalizumab in primarily non-Asian RRMS patients.

Enhanced content To view enhanced content for this article go to http://www.medengine.com/Redeem/ 8C47F060217D0440

T. Saida $(\bowtie)$

Kansai Multiple Sclerosis Center and Kyoto Min-iren

Central Hospital, Kyoto, Japan

e-mail: saida_takahiko@maia.eonet.ne.jp

J.-I. Kira

Department of Neurology, Graduate School of Medical Sciences, Kyushu University, Fukuoka, Japan

S. Kishida

Hakusuikai Hatsuishi Hospital, Chiba, Japan

T. Yamamura

National Center of Neurology and Psychiatry,

Kodaira, Tokyo, Japan

N. Ohtsuka $\cdot$ J. T. Tibung

Biogen Japan Ltd, Tokyo, Japan

Q. Dong

Biogen, Cambridge, MA, USA
Methods: This subanalysis of the placebo-controlled phase 2 trial of natalizumab in Japanese RRMS patients $(n=94)$ evaluated the effects of natalizumab versus placebo on the proportion of patients who achieved relapse-free, T1 gadolinium-enhancing $(\mathrm{Gd}+)$ lesion-free, and new/newly enlarged T2 lesion-free status, defined as "no evidence of inflammatory disease activity" (NEDA)-like status, after 24 weeks of treatment.

Results: In this subanalysis, significantly more natalizumab-treated than placebo-treated patients achieved NEDA-like status (76.6\% vs. $31.9 \% ; P<0.0001)$. In addition, the odds ratio (95\% confidence interval) for patients on natalizumab to reach NEDA-like status was 6.98 (2.80-17.38) compared with placebo patients.

Conclusion: These results confirm previous findings indicating that natalizumab is efficacious in Japanese patients with RRMS.

Funding: Biogen.Trial

Registration: ClinicalTrials.gov identifier, NCT01440101.

Keywords: Gadolinium-enhancing lesions; Japanese patients; Natalizumab; Relapsingremitting multiple sclerosis; T2 lesions 


\section{INTRODUCTION}

The efficacy of natalizumab has been demonstrated in two pivotal phase 3 studies of primarily non-Asian patients with relapsing-remitting multiple sclerosis (RRMS) $[1,2]$. In both studies, natalizumab reduced the frequency of relapses, mitigated the development of new brain lesions on magnetic resonance imaging (MRI) scans, and delayed the time to confirmed disability worsening relative to placebo. Recently, the results of a phase 2 study of natalizumab in Japanese patients with RRMS were reported, and treatment-related changes in relapses, brain lesions, and disability worsening were comparable to those from the phase 3 studies [3].

It has been demonstrated that the endpoint of "no evidence of disease activity" (NEDA) over 2 years, a composite measure of disease activity, including relapses, sustained Expanded Disability Status Scale (EDSS) score progression, and MRI activity, may predict long-term disability progression [4]. In a phase 3 study of natalizumab in non-Asian patients with RRMS, $37 \%$ of natalizumab-treated patients showed freedom from disease activity (i.e., no radiological or clinical disease activity) compared with $7 \%$ of placebo-treated patients $(P<0.0001)$ [5]. However, since it was unknown whether similar effects would be seen in Japanese patients, we conducted this subanalysis of the phase 2 trial in Japanese patients with RRMS to evaluate the effects of natalizumab versus placebo on the proportion of patients achieving relapse-free, T1 gadolinium-enhancing $(\mathrm{Gd}+)$ lesion-free, and new/newly enlarged T2 lesion-free status, defined as NEDA-like status.

\section{METHODS}

Japanese patients (aged 18-65 years) were eligible for the two-part phase 2 trial, if they had a diagnosis of RRMS (revised McDonald criteria) [6], $\geq 1$ exacerbation within the previous year, and, for inclusion in part B of the trial, an EDSS [7] score of 0.0-5.5. In part A of the study, 12 patients received open-label intravenous natalizumab $300 \mathrm{mg}$ every 4 weeks for 24 weeks. Part B was a double-blind study in which 94 patients were randomized to natalizumab $300 \mathrm{mg}$ or placebo every 4 weeks for 24 weeks (Fig. 1) [3]. This subanalysis pertains only to patients randomized in part B of the study. The methodology of the full study, along with its efficacy and safety data, has been previously published [3].

Clinical relapses were defined as new or recurrent neurological symptoms that were not associated with fever or infection and lasted for $\geq 24 \mathrm{~h}$. Brain MRI scans were performed with T1 (with and without gadolinium) and $\mathrm{T} 2$ sequences.

The proportions of patients who were free of the following events during part B of the study were evaluated: relapses during weeks 0-24; T1 $\mathrm{Gd}+$ lesions at week 24; new or newly enlarged T2 lesions at week 24; and relapses, T1 Gd+ lesions, and new/newly enlarged $\mathrm{T} 2$ lesions at week 24. Unadjusted odds ratios (ORs) with 95\% confidence intervals (CIs) were calculated to evaluate differences in the likelihood of achieving event-free status between treatment groups. For comparisons between treatment groups, $P$ values were based on Fisher exact tests.

\section{Compliance with Ethics Guidelines}

The study was conducted in accordance with Good Clinical Practice (GCP) guidelines, with applicable local regulations (including Japanese Ministry of Health, Labour and Welfare regulations), and with the Declaration of Helsinki of 1964, as revised in 2013. The protocol was approved by ethics committees at

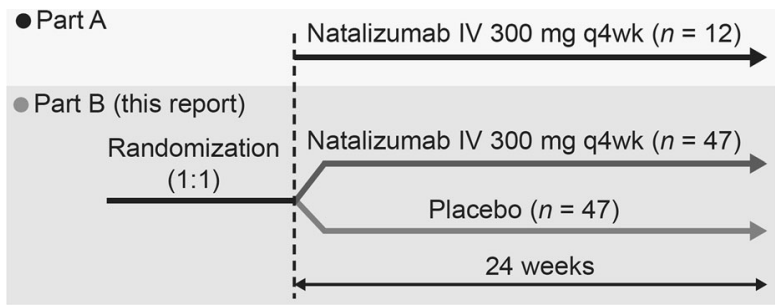

Fig. 1 Study flow diagram. $I V$ intravenous, $q 4 w k$ every 4 weeks 
all sites, and all patients provided written informed consent.

\section{RESULTS}

This subanalysis included data from all patients randomized to natalizumab $(n=47)$ or placebo $(n=47)$ in part B of the study. A significantly larger proportion of natalizumab patients $(76.6 \%)$ than placebo patients (31.9\%) achieved relapse-free, T1 Gd+ lesion-free, and new/newly enlarged T2 lesion-free status, or NEDA-like status, at week $24 \quad(P<0.0001)$. Figure 2 presents the percentages of patients with each type of event.

The likelihood of achieving relapse-free, T1 $\mathrm{Gd}+$ lesion-free, and new/newly enlarged T2 lesion-free status was significantly greater with natalizumab treatment than with placebo (OR [95\% CI], 6.98 [2.80-17.38]) (Table 1). Overall, natalizumab patients were seven times as likely as placebo patients to be free of any event. When the events were analyzed separately,



Fig. 2 Proportions of event-free patients at week $24 .{ }^{\mathrm{a}} P=0.0003 ;{ }^{\mathrm{b}} P=0.0002 ;{ }^{\mathrm{c}} P<0.0001 . G d+$ gadolinium-enhancing

Table 1 Likelihood of freedom from events for natalizumab versus placebo patients (at week 24)

\begin{tabular}{llr}
\hline Freedom from: & OR $(\mathbf{9 5 \%}$ CI $)$ & $\boldsymbol{P}$ value \\
\hline Relapse & $5.70(2.25,14.43)$ & 0.0003 \\
T1 Gd+ lesions & $9.95(2.69,36.76)$ & 0.0002 \\
New/newly enlarged T2 lesions & N/A & 0.0559 \\
NEDA-like status & $6.98(2.80,17.38)$ & $<0.0001$ \\
\hline
\end{tabular}

$C I$ confidence interval, $G d+$ gadolinium-enhancing, $N / A$ not applicable, $O R$ odds ratio

a The OR for being free of new/newly enlarged T2 lesions was not calculated because all natalizumab patients achieved new/newly enlarged T2 lesion-free status 
natalizumab patients were nearly six times as likely to be free of relapses and nearly ten times as likely to be free of $\mathrm{T} 1 \mathrm{Gd}+$ lesions as placebo patients at week 24 .

\section{DISCUSSION}

In this subanalysis of data from the phase 2 study of natalizumab in Japanese patients with RRMS, a significantly larger proportion of natalizumab patients than placebo patients reached NEDA-like status after 24 weeks of treatment.

Therapeutic efficacy can be difficult to demonstrate in multiple sclerosis (MS) clinical trials, as the currently accepted clinically relevant measures of disease activity are infrequent (e.g., relapses) and/or slow to develop (e.g., disability worsening) [8]. A post hoc analysis of data from AFFIRM (a 2-year, randomized, placebo-controlled phase 3 trial of RRMS patients $[N=942]$ ) was the first to include an assessment of individual outcomes as a composite measure: "freedom from disease activity", defined as no relapses, no 12-week-confirmed disability worsening, no $\mathrm{Gd}+$ lesions, and no new/newly enlarged T2-hyperintense lesions. In this trial, a larger proportion of natalizumab patients (37\%) than placebo patients (7\%) showed freedom from disease activity [5]. Similar composite endpoints have been evaluated in more recent MS clinical trials, though the terminology NEDA is becoming more frequently used [8].

Although the components and definitions of NEDA vary across studies [4], the proportion of natalizumab-treated patients who were free of relapses, T1 Gd+ lesions, and new/newly enlarged T2 lesions in the current analysis $(77 \%)$ was comparable to that in previous reports of natalizumab treatment in Italian RRMS patients and in European, North American, Australian, and New Zealand patients with relapsing MS from the AFFIRM trial $(37-87 \%)[5,9]$. The current study differs from the AFFIRM trial in that the proportion of patients free from new/newly enlarged T2 lesions in this study was not significantly larger with natalizumab than placebo. In addition, a similar result was observed in changes of mean EDSS score, referentially collected, in both groups (baseline score: placebo 2.05 vs. natalizumab 2.45; and score at 24 weeks: placebo 2.16 vs. natalizumab 2.25) [3]. These may be due, at least in part, to the shorter duration of this study (24 weeks) compared with AFFIRM (2 years) [5].

Aside from studies of natalizumab, other disease-modifying therapies (DMTs) studies from around the world have assessed the proportions of patients achieving freedom from disease activity [10-13]. Other trials have examined the efficacy of certain DMTs (e.g., interferon beta- $1 \mathrm{a}$, interferon beta- $1 \mathrm{~b}$, and fingolimod) exclusively in Japanese patients [14-16], though head-to-head comparator trials of the efficacy of DMTs in Japanese patients have not yet been conducted nor have NEDA data of other DMTs in Japanese patients been published. However, NEDA results are available in studies conducted mainly in Caucasian patients with MS (e.g., after 2 years of follow-up, NEDA-3 was reported in $70 \%$ of natalizumab treated patients vs. $44 \%$ of fingolimod treated patients) [17].

Our findings should be interpreted in light of some limitations. The number of patients evaluated was relatively small, and the 24-week study period was relatively short. Evidence suggests that EDSS worsening confirmed at $\geq 24$ weeks is a meaningful intermediate clinical outcome measure that predicts clinically significant disability [18]; however, confirmed EDSS worsening, which is often a component of NEDA, was not incorporated into the composite endpoint in this analysis due to the short study period.

\section{CONCLUSION}

In conclusion, this subanalysis of data from the Japanese phase 2 study of natalizumab in RRMS patients demonstrates that a significantly larger percentage of natalizumab patients than placebo patients were free of relapses, T1 Gd+ lesions, and new/newly enlarged T2 lesions. These results are consistent with findings from non-Asian patient populations $[1,2]$ and 
support the efficacy of natalizumab in Japanese patients with RRMS.

\section{ACKNOWLEDGEMENTS}

Biogen provided funding for this study, for the publication of this article, and for editorial support in the development of this manuscript; Linda A. Goldstein, PhD, and Alexandra W. Davis (Ashfield Healthcare Communications, Middletown, CT, USA) provided writing support based on input from authors, and Joshua Safran (Ashfield Healthcare Communications) copyedited and styled the manuscript per journal requirements. This study was supported by Biogen (Cambridge, MA, USA), which provided funding for editorial support in the development of this paper and reviewed and provided feedback on the paper to the authors. The authors had full editorial control of the paper and provided their final approval of all content. All authors had full access to all of the data in this study and take complete responsibility for the integrity of the data, the accuracy of the data analysis, and the work as a whole. All named authors meet the International Committee of Medical Journal Editors (ICMJE) criteria for authorship for this manuscript, take responsibility for the integrity of the work as a whole, and have given final approval for the version to be published. Portions of this manuscript were submitted as an abstract to the 25th Annual Meeting of the Japanese Society for Neuroimmunology; this abstract was accepted for publication and was presented as a poster on September 29-30, 2016, in Nagasaki, Japan.

Disclosures. Takahiko Saida (a coordinating investigator) has received funding from, held board membership for, spoken at scientific meetings for, prepared manuscripts for, and had consulting agreements with Astellas, Biogen, Bayer-Schering, Daiichi-Sankyo, Eisai, Kaketsuken, Merck Serono, Mitsubishi-Tanabe, Nihon, Novartis, Ono, Sanofi, TDS Japan, and Teijin. Jun-ichi Kira (a coordinating investigator) has been a consultant for Biogen and Novartis and has received honoraria from Bayer Healthcare, Biogen, Boehringer Ingelheim, Eisai, Kyowa Kirin, Mitsubishi Tanabe, Otsuka, Pfizer Japan, and Teijin and funding for travel from Bayer HealthCare and Biogen, as well as grants from the Japanese Ministry of Health, Labour and Welfare, the Japanese Science and Technology Agency, and the Japanese Ministry of Education, Culture, Sports, Science and Technology. Shuji Kishida (the PML consultant) has been a consultant for Ajinomoto. Takashi Yamamura (the scientific advisor) has been a member of scientific advisory boards for Biogen and Chugai; received research support from Asahi Kasei Medical, Chugai, Mitsubishi Tanabe, Ono, and Teva; received speaker honoraria from Abbot Japan, Astellas, Bayer, Biogen, Dainippon Sumitomo, Eisai, Mitsubishi Tanabe, Nihon, Novartis, and Santen; and been funded by the Japanese Ministry of Health, Labour and Welfare and the Japan Society for the Promotion of Science. Nobuhisa Ohtsuka was an employee of Biogen at the time this analysis was performed. Qunming Dong is an employee of and holds stock and/or stock options in Biogen. J. T. Tibung is an employee of and holds stock and/or stock options in Biogen.

Compliance with Ethics Guidelines. The study was conducted in accordance with Good Clinical Practice (GCP) guidelines, with applicable local regulations (including Japanese Ministry of Health, Labour and Welfare regulations), and with the Declaration of Helsinki of 1964, as revised in 2013. The protocol was approved by ethics committees at all sites, and all patients provided written informed consent.

Data Availability. The datasets generated and/or analyzed during the current study are not publicly available and are fully owned by Biogen, but are available from Nisha Lucas at nisha.lucas@biogen.com on reasonable request.

Open Access. This article is distributed under the terms of the Creative Commons Attribution-NonCommercial 4.0 International License (http://creativecommons.org/licenses/ by-nc/4.0/), which permits any noncommercial use, distribution, and reproduction in any 
medium, provided you give appropriate credit to the original author(s) and the source, provide a link to the Creative Commons license, and indicate if changes were made.

\section{REFERENCES}

1. Polman $\mathrm{CH}, \mathrm{O}^{\prime}$ Connor PW, Havrdova E, Hutchinson M, Kappos L, Miller DH, et al. A randomized, placebo-controlled trial of natalizumab for relapsing multiple sclerosis. N Engl J Med. 2006;354(9):899-910.

2. Rudick RA, Stuart WH, Calabresi PA, Confavreux C, Galetta SL, Radue EW, et al. Natalizumab plus interferon beta-1a for relapsing multiple sclerosis. N Engl J Med. 2006;354(9):911-23.

3. Saida T, Kira J, Kishida S, Yamamura T, Sudo Y, Ogiwara $\mathrm{K}$, et al. Efficacy, safety, and pharmacokinetics of natalizumab in Japanese multiple sclerosis patients: a doubleblind, randomized controlled trial and open-label pharmacokinetic study. Mult Scler Relat Disord. 2017;11(1):25-31.

4. Rotstein DL, Healy BC, Malik MT, Chitnis T, Weiner HL. Evaluation of no evidence of disease activity in a 7-year longitudinal multiple sclerosis cohort. JAMA Neurol. 2015;72(2):152-8.

5. Havrdova E, Galetta S, Hutchinson M, Stefoski D, Bates D, Polman $\mathrm{CH}$, et al. Effect of natalizumab on clinical and radiological disease activity in multiple sclerosis: a retrospective analysis of the Natalizumab Safety and Efficacy in Relapsing-Remitting Multiple Sclerosis (AFFIRM) study. Lancet Neurol. 2009;8(3):254-60.

6. Polman $\mathrm{CH}$, Reingold SC, Edan G, Filippi M, Hartung HP, Kappos L, et al. Diagnostic criteria for multiple sclerosis: 2005 revisions to the "McDonald Criteria". Ann Neurol. 2005;58(6):840-6.

7. Kurtzke JF. Rating neurologic impairment in multiple sclerosis: an expanded disability status scale (EDSS). Neurology. 1983;33(11):1444-52.

8. Bevan CJ, Cree BA. Disease activity free status: a new end point for a new era in multiple sclerosis clinical research? JAMA Neurol. 2014;71(3):269-70.

9. Totaro R, Lugaresi A, Bellantonio P, Danni M, Costantino G, Gasperini C, et al. Natalizumab treatment in multiple sclerosis patients: a multicenter experience in clinical practice in Italy.
Int J Immunopathol Pharmacol. 2014;27(2):147-54.

10. Giovannoni G, Cook S, Rammohan K, Rieckmann $\mathrm{P}$, Sorensen PS, Vermersch P, et al. Sustained disease-activity-free status in patients with relapsing-remitting multiple sclerosis treated with cladribine tablets in the CLARITY study: a post hoc and subgroup analysis. Lancet Neurol. 2011;10(4):329-37.

11. Havrdova E, Gold R, Fox R, Kappos L, Phillips J, Zhang $A$, et al. BG-12 (dimethyl fumarate) treatment for relapsing-remitting multiple sclerosis (RRMS) increases the proportion of patients free of measured clinical and neuroradiologic disease activity in the phase 3 studies. The American Academy of Neurology 65th Annual Meeting, March 16-23, 2013, San Diego, CA.

12. Khatri B, Barkhof F, Comi G, Jin J, Francis G, Cohen J. Fingolimod treatment increases the proportion of patients who are free from disease activity in multiple sclerosis compared to interferon beta-1a: results from a phase 3 active-controlled study (TRANSFORMS). The American Academy of Neurology 64th Annual Meeting, April 21-28, 2012, New Orleans, LA.

13. Barkhof F, Cohen J, Radue E, Kappos L, Calabresi P, Häring D, et al. Brain volume changes, on-study correlations and the link to disability in three fingolimod phase 3 studies. The 29th Congress of the European Committee for Treatment and Research in Multiple Sclerosis, October 2-5, 2013, Copenhagen, Denmark.

14. Saida $\mathrm{T}$, Kira J, Ueno $\mathrm{Y}$, Harada $\mathrm{N}$, Hirakata $\mathrm{T}$. Long-term efficacy and safety of intramuscular interferon beta-1a: randomized postmarketing trial of two dosing regimens in Japanese patients with relapsing-remitting multiple sclerosis. Mult Scler Relat Disord. 2016;7:102-8.

15. Saida $T$, Tashiro K, Itoyama $Y$, Sato $T$, Ohashi $Y$, Zhao $\mathrm{Z}$, et al. Interferon beta- $1 \mathrm{~b}$ is effective in Japanese RRMS patients: a randomized, multicenter study. Neurology. 2005;64(4):621-30.

16. Saida T, Kikuchi S, Itoyama $\mathrm{Y}, \mathrm{Hao} Q$, Kurosawa T, Nagato K, et al. A randomized, controlled trial of fingolimod (FTY720) in Japanese patients with multiple sclerosis. Mult Scler. 2012;18(9):1269-77.

17. Baroncini D, Ghezzi A, Annovazzi PO, Colombo B, Martinelli V, Minonzio G, et al. Natalizumab versus fingolimod in patients with relapsing-remitting multiple sclerosis non-responding to first-line injectable therapies. Mult Scler. 2016;22(10):1315-26. 
18. Rudick RA, Lee JC, Cutter GR, Miller DM, Bourdette D, Weinstock-Guttman B, et al. Disability progression in a clinical trial of relapsing-remitting multiple sclerosis: eight- year follow-up. Arch Neurol. 2010;67(11): 1329-35. 